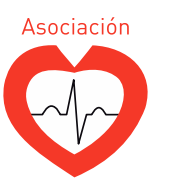

SOCIEDAD COLOMBIANA DE CARDIOLOGÍA \& CIRUGÍA CARDIOVASCULAR

\section{Revista Colombiana de \\ Cardiología \\ www.elsevier.es/revcolcar}

CARDIOLOGÍA DEL ADULTO - ARTÍCULO ORIGINAL

\title{
Comparación de las características y curso clínico de la endocarditis infecciosa por Staphylococcus aureus meticilino sensible versus meticilino resistente
}

\author{
Fernando Rosso ${ }^{\mathrm{a}, *}$, Jorge Andrés Cedano ${ }^{\mathrm{b}}$, \\ María Alejandra Franco-Molina ${ }^{c}$, Andrés Felipe Borrero-González ${ }^{c}$ \\ y Juan Esteban García-Robledoc
}

\author{
a Servicio de Infectología, Fundación Valle del Lili, Cali, Colombia

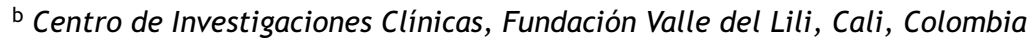 \\ c Medicina, Universidad Icesi, Cali, Colombia
}

Recibido el 25 de julio de 2017; aceptado el 26 de marzo de 2018

Disponible en Internet el 8 de junio de 2018

\author{
PALABRAS CLAVE \\ Endocarditis; \\ Meticilina; \\ Mortalidad; \\ Estafilococo; \\ Embolización
}

\begin{abstract}
Resumen
Introducción: Staphylococcus aureus es uno de los agentes causales más comunes de la endocarditis infecciosa. Se reportan pocos estudios en Latinoamérica acerca de las diferencias entre los perfiles de resistencia a la meticilina.

Objetivo: Describir las características y el curso clínico de los pacientes con S. aureus sensible a meticilina frente al resistente.

Métodos: Estudio observacional retrospectivo, cohorte histórica de pacientes adultos con diagnóstico confirmado de endocarditis entre los años 2011 y 2015. Se seleccionaron pacientes positivos para $S$. aureus comparando las características y el curso clínico entre los casos $S$. aureus sensible a meticilina frente al resistente.

Resultados: Se estudiaron 86 pacientes con endocarditis. 28 (33\%) tenían infección por S. aureus. $21(75 \%)$ tenían endocarditis por S. aureus sensible a meticilina y $7(25 \%)$ por S. aureus resistente a meticilina. En el grupo S. aureus sensible a meticilina, 11 $(52,3 \%)$ fueron infecciones asociadas a atención en salud. La mayoría de casos de $S$. aureus resistente a meticilina fueron $(85,7 \%)$ adquiridos en comunidad. La mortalidad de endocarditis por $S$. aureus sensible a meticilina fue superior a la causada por el resistente (33,3\% vs. $14 \%)$.
\end{abstract}

\footnotetext{
* Autor para correspondencia.

Correo electrónico: frosso07@gmail.com (F. Rosso).
} 
Conclusiones: S. aureus sigue siendo el agente más frecuente en endocarditis, más comúnmente el sensible a la meticilina. Los eventos embólicos y la gravedad fueron mayores en $S$. aureus sensible a meticilina. La mayor proporción de endocarditis debido a $S$. aureus resistente a meticilina se adquirió en la comunidad, por lo que se sugiere iniciar cobertura empírica contra S. aureus resistente a meticilina en todo caso de endocarditis adquirida en la comunidad.

(c) 2018 Sociedad Colombiana de Cardiología y Cirugía Cardiovascular. Publicado por Elsevier España, S.L.U. Este es un artículo Open Access bajo la licencia CC BY-NC-ND (http:// creativecommons.org/licenses/by-nc-nd/4.0/).

\section{KEYWORDS}

Endocarditis; Methicillin; Mortality; Staphylococcus; Embolisation

\section{Characteristics and clinical course of endocarditis due to Methicillin-sensitive versus methicillin-resistant Staphylococcus aureus}

\begin{abstract}
Introduction: Staphylococcus aureus is one of the most common sources of infectious endocarditis. There are few studies in Latin America that report on the differences between the methicillin resistance profiles.

Objective: To describe the characteristics and clinical course of patients with methicillinsensitive S. aureus (MSSA) compared to methicillin-resistance S. aureus (MRSA)

Methods: An observational, retrospective study was conducted on a historical cohort of adult patients with a confirmed diagnosis of endocarditis between the years 2011 and 2015. Patients positive for $S$. aureus were selected and the characteristics and clinical course and the cases of MSSA were compared with those of MRSA.

Results: A total of 86 patients with endocarditis were included, of whom 28 (33\%) had an infection due to $S$. aureus, and 21 (75\%) had endocarditis due to methicillin-sensitive S. aureus, and $7(25 \%)$ due to MRSA. In the MSSA group, $11(52.3 \%)$ were infections associated with health care. The majority $(85.7 \%)$ of cases of MRSA were community acquired. The endocarditis mortality due to MSSA was higher than that caused by MRSA (33.3\% vs. $14 \%)$.

Conclusions: S. aureus continues to be the most common agent in endocarditis, with MSSA being more common. The embolic events and the severity were greater in MSSA. The majority of endocarditis due to MRSA is acquired in the community, and for this reason it is suggested starting empirical cover against MRSA in all cases of community acquired endocarditis.

(C) 2018 Sociedad Colombiana de Cardiología y Cirugía Cardiovascular. Published by Elsevier España, S.L.U. This is an open access article under the CC BY-NC-ND license (http:// creativecommons.org/licenses/by-nc-nd/4.0/).
\end{abstract}

\section{Introducción}

La endocarditis infecciosa es una infección de la superficie endocárdica, que usualmente compromete las válvulas y/o los dispositivos intracardiacos. En países desarrollados se reportan incidencias anuales entre 3-9 casos por 100.000 habitantes $^{1}$, con una mortalidad que varía entre 15\%-22\% ${ }^{2}$. En Colombia se carece de información sobre este tema, con solo algunos estudios que reportan índices similares de mortalidad a los de países industrializados ${ }^{3}$, aunque faltan datos precisos de su incidencia en el país.

A pesar de los avances en el diagnóstico y tratamiento de esta patología, la frecuencia de la enfermedad ha disminuido pero no ha sido controlada. Incluso en países desarrollados, se registra un aumento de casos en pacientes con válvulas protésicas, dispositivos intracardiacos, enfermedad cardiaca cianosante o en pacientes con endocarditis infecciosa previa ${ }^{1}$. En la actualidad la valvulopatía reumática es cada vez menos frecuente en países desarrollados, aunque no tanto en países latinoamericanos, lo cual ha sido descrito en investigaciones nacionales ${ }^{1,3,4}$.
Staphylococcus aureus es el agente causal más reportado en la actualidad, correspondiendo al $31 \%$ de casos en algunos estudios. Se han descrito múltiples factores a nivel del hospedero que predisponen al desarrollo de endocarditis infecciosa por este microorganismo, entre los que figuran el uso de drogas endovenosas, tratamientos intrahospitalarios invasivos, especialmente aquellos que impliquen la presencia de dispositivos intracardiacos, ser portador de válvulas protésicas, requerimiento de hemodiálisis, entre otros. Adicionalmente, el $\mathrm{S}$. aureus está asociado con mayores grados de severidad clínica de la endocarditis infecciosa ${ }^{1,5-8}$. Este microorganismo tiene diferentes perfiles de resistencia, entre los que destacan: sensibilidad a meticilina (SAMS), resistente a meticilina (SAMR), y resistencia intermedia a vancomicina y a vancomicina. De los anteriores perfiles, SAMS y SAMR son los más comúnmente asociados a endocarditis infecciosa?.

En múltiples estudios se han evaluado las características clínicas de las infecciones por $S$. aureus en la endocarditis infecciosa sin tener en cuenta los perfiles de resistencia. Por consiguiente existe poca información sobre las diferencias respecto a mortalidad, complicaciones y factores de riesgo 


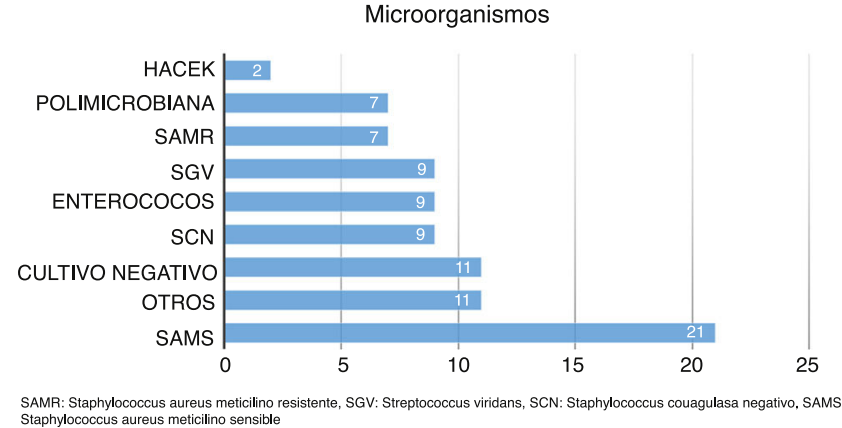

Figura 1 Microorganismos causantes más frecuentes en endocarditis infecciosa.

que existen entre SAMS y SAMR. Ciertas investigaciones en Europa y Norteamérica han evaluado esta relación, pero en Colombia la información sobre las diferencias entre estos dos organismos en endocarditis infecciosa es escasa.

\section{Métodos}

Estudio descriptivo, retrospectivo, llevado a cabo en la Fundación Valle del Lili, centro hospitalario de cuarto nivel, en Cali, Colombia. Se incluyeron pacientes desde enero de 2011 hasta diciembre de 2015. La información se obtuvo de la historia clínica y de resultados de pruebas de laboratorio de microbiología. La información se registró y organizó en una base de datos y un formato de reporte de caso electrónico.

Se incluyeron pacientes hospitalizados con diagnóstico CIE-10 de endocarditis en adultos que tuvieran diagnóstico microbiológico y ecocardiográfico de endocarditis realizado en la institución. Se excluyeron pacientes sin información suficiente en la historia clínica, o falta de disponibilidad de las imágenes cardíacas, sin resultados microbiológicos. Se re-analizaron los casos utilizando las criterios de Duke, para aumentar la probabilidad de endocarditis. Una vez verificados los criterios de inclusión y exclusión, se extrajo la información demográfica, clínica y ecocardiográfica de sus historias clínicas, así como los resultados de cultivos y perfiles de sensibilidad del laboratorio de microbiología. De la base de datos global, se identificaron los pacientes con infección por $S$. aureus y se clasificaron según los perfiles de resistencia a la meticilina en endocarditis infecciosa causada por SAMS o SAMR.

Las variables categóricas se analizaron por medio de chicuadrado test o test de Fisher. Además, a las variables de los participantes se les realizó un análisis univariado en el que se incluyó edad, género, mortalidad, complicaciones, sitio afectado, entre otros. Se determinó el nivel significancia de $p$ en $<0,05$. Finalmente, el análisis estadístico se procesó en STATA (Statistics and Data software StataCorp 1996-2017) versión 13.

\section{Resultados}

Se estudiaron 86 individuos con diagnóstico de endocarditis infecciosa entre 2011 y 2015. Como agentes causales de la enfermedad, con mayor frecuencia se hallaron cocos gram positivos, seguidos por bacilos gram negativos (fig. 1). El germen más aislado fue $S$. aureus, con $33 \% \quad(n=28)$, seguido de Enterococcus spp, Staphylococcus coagulasa negativo y Streptococcus del grupo viridans, cada uno con $11 \%$. En cuanto a la distribución de perfiles de resistencia del S. aureus, se encontró que $75 \%(n=21)$ eran SAMS y $25 \%$ $(\mathrm{n}=7)$ eran SAMR (tabla 1$)$.

Una vez separados, se compararon las diferencias entre SAMS y SAMR. No se identificaron diferencias estadísticamente significativas para las variables estudiadas. La edad de los pacientes fue 59 años para el SAMS y 55 años para el SAMR $(p=0,542)$. La endocarditis infecciosa fue más frecuente en hombres (SAMS 52,4\% y SAMR 85,7\%; $p=0,191$ ). No se identificaron diferencias significativas en ninguna comorbilidad analizada (tabla 2). Cabe resaltar que no se encontraron explícitos en las historias clínicas datos sobre el antecedente de uso de drogas endovenosas, en ninguno de los casos de endocarditis infecciosa por S. aureus.

Al evaluar la distribución entre infecciones asociadas a la atención en salud (IAAS) y las adquiridas en la comunidad (IAC), se encontró que de los 21 casos de endocarditis infecciosa por SAMS, $11(52,4 \%)$ fueron IAAS, mientras que de los 7 casos por SAMR, solo $1(14,3 \%)$ fue IAAS $(p=0,091)$. El resto de casos correspondió a IAC (tabla 2). En cuanto al tiempo de estancia hospitalaria, el promedio en días para los infectados por SAMS fue de 37,7 días, mientras que para SAMR fue de 37 días $(p=0,945)$. Por otro lado, tanto en los casos de SAMS como SAMR, el $85,7 \%$ de los pacientes $(p=1,0)$ requirió manejo en unidad de cuidado intensivo (UCI) (tabla 2). En cuanto a mortalidad, aunque tampoco hubo significancia estadística $(p=0,633)$, fallecieron 7 pacientes con infección por SAMS $(33,3 \%)$ y 1 por SAMR $(14,3 \%)$.

En lo referente a las complicaciones asociadas a estos dos microorganismos, no se encontraron diferencias significativas en las variables estudiadas, pero cabe resaltar que ocurrió insuficiencia valvular en 15 de los casos por SAMS y en todos los casos por SAMR $(71,4 \%$ vs. $100 \%$; $p=0,366)$ y que los eventos embólicos fueron más comunes en la endocarditis infecciosa por SAMS que por SAMR $(66,7 \%$ vs. $28,6 \%$; $p=0,103$ ) (tabla 2).

Además, no se halló predominio de endocarditis infecciosa de lado izquierdo o derecho en los casos por SAMS $(47,6 \%$, respectivamente; $p=1,0)$, mientras que en los pacientes con SAMR hubo un ligero predominio de endocarditis infecciosa izquierda ( $57 \%$ vs. $43 \% ; p=1,0)$ (tabla 2 ). Finalmente, se analizó la relación según el tipo de válvula nativa y protésica, en cuyo caso no se encontró diferencia significativa entre SAMS y SAMR (tabla 2).

\section{Discusión}

Históricamente el S. aureus ha sido el germen más reportado en endocarditis infecciosa; varias series mundiales muestran tasas de $24 \%$ a $51 \%^{3,4,7,9}$, mientras estudios nacionales reportan incidencias del $13 \%{ }^{10}$. En este estudio se hallaron incidencias similares, las cuales se asemejan más a lo reportado por la literatura global. Por otro lado, fue más común el aislamiento de SAMS que de SAMR, hecho que también es semejante a lo descrito en Colombia y otras regiones del mundo $0^{3,7,11,12}$.

Es llamativo que, según esta investigación, los casos de endocarditis infecciosa por ambas cepas de S. aureus son más jóvenes que lo reportado en otros países. 
Tabla 1 Características y antecedentes predisponentes

\begin{tabular}{llll}
\hline & SAMS n=21 \# (\%) & SAMR n=7 \# (\%) & Valor $\mathrm{p}$ \\
\hline $\begin{array}{l}\text { Características } \\
\text { Edad media ( } \pm \mathrm{DE})\end{array}$ & $59,8( \pm 16,6)$ & $55( \pm 21,3)$ & 0,529 \\
Masculino & $11(52,4)$ & $6(85,7)$ & 0,191 \\
Antecedentes & & & \\
Fiebre reumática & $0(0)$ & $1(14.3)$ & 0.25 \\
Tabaquismo & $4(19)$ & $2(28.6)$ & 0,606 \\
Enfermedad periodontal & $3(14,3)$ & $1(14,3)$ & 1 \\
Defecto valvular previo & $7(33,3)$ & $3(42,9)$ & 0,674 \\
ICC & $8(38,1)$ & $2(28,6)$ & 1 \\
SCA & $3(14,3)$ & $0(0)$ & 0,551 \\
HTA & $14(66,7)$ & $4(57,1)$ & 0,649 \\
ECV & $4(19)$ & $1(14,3)$ & 1 \\
Enfermedad reumatológica & $3(14,3)$ & $0(0)$ & 0,551 \\
Endocarditis infecciosa & $1(4,8)$ & $2(28,6)$ & 0,145 \\
IRC & $11(52,4)$ & $2(28,6)$ & 0,396 \\
Cirrosis hepática & $1(4,8)$ & $0(0)$ & 1 \\
Malignidad & $5(23,8)$ & $3(42,9)$ & 0,371 \\
DM & $7(33,3)$ & $3(42,9)$ & 0,649 \\
Esteroides & $3(14,3)$ & $1(14,3)$ & 1 \\
\hline
\end{tabular}

ICC: insuficiencia cardiaca congestiva, SCA: síndrome coronario agudo, HTA: hipertensión arterial, ECV: evento cerebrovascular, El: endocarditis infecciosa, ICR: falla renal aguda, DM: diabetes mellitus.

Tabla 2 Características valvulares y desenlaces clínicos

\begin{tabular}{|c|c|c|c|}
\hline & $\begin{array}{l}\text { SAMS n=21 } \\
\#(\%)\end{array}$ & $\begin{array}{l}\text { SAMR } \mathrm{n}=7 \\
\#(\%)\end{array}$ & Valor $\mathrm{p}$ \\
\hline \multicolumn{4}{|l|}{ Sitio afectado } \\
\hline Izquierda & $10(47,6)$ & $4(57,1)$ & 1 \\
\hline Derecha & $10(47,6)$ & $3(42,9)$ & 1 \\
\hline SD & $1(4,8)$ & $0(0)$ & \\
\hline \multicolumn{4}{|l|}{ Origen de la infección } \\
\hline IAAS & $11(52,4)$ & $1(14,3)$ & 0,091 \\
\hline IAC & $10(47,6)$ & $6(85,7)$ & 0,184 \\
\hline \multicolumn{4}{|l|}{ Complicaciones } \\
\hline ICC & $12(57,1)$ & $3(42,9)$ & 0,67 \\
\hline Artritis séptica & $1(4,8)$ & $0(0)$ & 1 \\
\hline Ruptura valvular & $3(14,3)$ & $2(28,6)$ & 0,574 \\
\hline Insuficiencia valvular & $15(71,4)$ & $7(100)$ & 0,366 \\
\hline Eventos embólicos & $14(66,7)$ & $2(28,6)$ & 0,103 \\
\hline \multicolumn{4}{|l|}{ Desenlaces clínicos } \\
\hline Días de estancia $( \pm \mathrm{DE})$ & $37,7( \pm 25,5)$ & $37( \pm 15,5)$ & 0,945 \\
\hline $\mathrm{UCl}$ & $18(85,7)$ & $6(85,7)$ & 1 \\
\hline Requerimiento quirúrgico & $9(42,9)$ & $2(28,6)$ & 0,668 \\
\hline Mortalidad & $7(33,3)$ & $1(14,30$ & 0,633 \\
\hline \multicolumn{4}{|l|}{ Característica valvular } \\
\hline Nativa & 6 & 16 & 1 \\
\hline Protésica & 1 & 2 & 1 \\
\hline Dispositivo & 0 & 3 & 0,5 \\
\hline
\end{tabular}

IAAS: infecciones asociadas a la atención en salud; IAC: infecciones adquiridas en la comunidad; UCI: unidad de cuidados intensivos, SD: sin dato. 
En Bélgica, Hill et $\mathrm{al}^{7}$. documentaron que los pacientes con endocarditis infecciosa por SAMR tenían edades más avanzadas que aquellos que padecían infecciones por SAMS, contrario a lo que se encontró en este estudio, que muestra mayor edad en pacientes con SAMS. Estos datos requieren mayor número de muestra para lograr obtener una conclusión más precisa sobre estas características en países Latinoamericanos.

Respecto a otros factores de riesgo que predisponen al desarrollo de endocarditis infecciosa, aproximadamente una tercera parte de las endocarditis infecciosas por S. aureus se asociaron a un defecto valvular previo, siendo más común en infecciones por SAMR, lo cual es similar a lo reportado en la literatura mundial ${ }^{1,3,4}$. Sin embargo, cabe resaltar que la frecuencia de los defectos valvulares previos fue menor en nuestra población de estudio que lo reportado por otros autores ${ }^{7}$.

En cuanto a los antecedentes, se encontró mayor proporción de endocarditis previa en pacientes con SAMR, hecho que es contrario a lo reportado en estudios mundiales ${ }^{7}$; se destaca que los valores no fueron estadísticamente significativos. No se encontraron factores de riesgo claros que predispusieran a infecciones por los diferentes perfiles de resistencia, pero hacen falta estudios con mayor número poblacional y seguimientos prospectivos para llegar a una conclusión adecuada.

En este estudio sólo un caso de endocarditis infecciosa por SAMR presentaba antecedente de catéter intravascular, mientras que más de la mitad de los casos de endocarditis infecciosa por SAMS se asociaron a este factor de riesgo. En la literatura se ha descrito que si bien la presencia de catéteres endovasculares es uno de los principales factores de riesgo para desarrollar endocarditis infecciosa por $S$. aureus $s^{1,7,8,12,13}$, este se describe con mayor frecuencia en las infecciones por SAMR que por SAMS ${ }^{7}$, de manera que debería continuar evaluándose esta variable en estudios posteriores. Así mismo, no se logró documentar la presencia o no del antecedente de uso de drogas endovenosas en ninguna de las historias de los casos de endocarditis infecciosa por S. aureus, que es otro de los factores de riesgo más descritos para bacteriemias y endocarditis infecciosa por este agente 1,3-5,12,13. $^{1}$.

Cuando se analizó la lateralidad del compromiso valvular se observó que la endocarditis infecciosa por SAMS afectó en igual proporción las cavidades izquierdas y derechas, mientras que en los casos de SAMR existió predominio de la endocarditis infecciosa de cavidades izquierdas, sin ser relevante la diferencia. Este hallazgo es llamativo puesto que en la literatura el compromiso de cavidades izquierdas tiende a sobrepasar al de las cavidades derechas sin importar el germen involucrado $3,4,8,11,14$, tendencia que se mantiene al estudiar la endocarditis infecciosa por $S$. aureus en general ${ }^{6,7,12}$. En un estudio realizado en Korea por Hee et $\mathrm{al}^{15}$. se encontró que el compromiso de cavidades izquierdas fue más común para endocarditis infecciosa por SAMS, pero en los casos de infecciones por SAMR fue más común la afectación de cavidades derechas, datos que no pudieron encontrarse en este estudio. Finalmente, estudios en Europa muestran que el SAMS afecta más las válvulas nativas mientras SAMR no tenía predilección ${ }^{7}$, lo cual se correlaciona con lo encontrado en este estudio, que muestra mayor compromiso de válvula nativa, pero no solo en SAMS sino también en SAMR.

El SAMS está generalmente asociado a infecciones adquiridas en la comunidad, que en contraste con los hallazgos de este estudio se encontró que no existió diferencia entre IAAS e IAC $1,3,7,11,15$. Adicionalmente, es llamativo que las IAAS fueron más frecuentes en los casos de SAMS que de SAMR, dato importante ya que SAMR es un microrganismo que se asocia históricamente a IAAS ${ }^{7,12,16}$. En esta investigación se determinó que las endocarditis infecciosas por SAMR fueron mayoritariamente adquiridas en comunidad, dato que se relaciona con estudios en Brasil que muestran un posible cambio en el origen de este organismo, y que proponen que el SAMR proveniente de la comunidad está en aumento ${ }^{17}$. Es importante realizar más estudios, con mayor muestra poblacional, que permitan concluir la diferencia que puede existir según los perfiles de resistencia y el origen de la infección en países como Colombia.

En cuanto a las complicaciones, tanto en la endocarditis infecciosa por SAMS como por SAMR, la insuficiencia valvular fue la complicación más común, especialmente para los casos por SAMR, en los que se presentó en la totalidad. Este hallazgo es compatible con lo reportado en la literatura, que muestra que estos gérmenes comprometen constantemente la función valvular ${ }^{1,13,18,19}$. En cuanto a los eventos embólicos, se halló mayor frecuencia en pacientes con infección por SAMS que por SAMR, hecho que concuerda con estudios desarrollados en Bélgica ${ }^{7}$, que concluyen que la embolia fue más frecuente en pacientes con SAMS con una diferencia significativa frente a SAMR.

Concerniente a los desenlaces clínicos, los días de estancia hospitalaria fueron similares en ambos grupos, así como el requerimiento de manejo en una $\mathrm{UCl}$, el cual fue necesario en más del $80 \%$ de los casos. Por otro lado, cerca de la mitad de los pacientes con endocarditis infecciosa por SAMS requirió manejo quirúrgico, mientras que menos del $30 \%$ con endocarditis infecciosa por SAMR tuvo que ser llevado a recambio valvular, hallazgos que concuerdan con otros estudios que comparan estos dos grupos ${ }^{7}$.

Estudios en Harvard ${ }^{20}$, muestran mayor mortalidad en pacientes con bacteriemia por SAMR en comparación con SAMS con un OR de 1,93, pero la base de la investigación no era el estudio de endocarditis. En este estudio se encontró mayor mortalidad en los casos por SAMS que en los casos por SAMR, contrario a los datos reportados por otros estudios ${ }^{7,15}$; cabe resaltar que la diferencia de mortalidad no fue estadísticamente significativa, por lo que se requieren más estudios con mayor muestra poblacional para evaluar las diferencias de mortalidad entre estos dos perfiles de resistencia en países Latinoamericanos.

Es importante aclarar que una de las mayores limitaciones de este estudio fue el tamaño de muestra final de episodios de endocarditis infecciosa por SA. El número de pacientes en cada grupo de sensible y resistentes era muy bajo, lo cual no confiere un poder estadístico suficiente. Aun así, es la serie de más casos reportada en Colombia en los últimos cinco años, en la que se diferencian los casos por susceptibilidad a la meticilina.

Como comentario final, llama la atención la proporción de pacientes con SAMR adquirido en la comunidad. Este fenómeno ha sido creciente en Colombia desde su 
descripción en 2006, con una distribución amplia en varias ciudades capitales del país ${ }^{21}$. Esto refuerza la necesidad de cobertura empírica contra SAMR en todo caso de endocarditis infecciosa adquirida en la comunidad en tanto se descarte este microorganismo. En otras series de manejo de bacteriemia por SA sin endocarditis infecciosa, el uso tardío de tratamiento antibiótico contra SAMR se asoció a mayor mortalidad $^{22}$.

\section{Conclusiones}

El Staphylococcus aureus fue el germen que más se reportó en nuestro estudio, así como en diferentes estudios en el mundo, siendo en general el SAMS el más comúnmente aislado. No se pudieron establecer factores de riesgo claros para la infección por SAMS versus SAMR, pero se identificaron resultados similares en cuanto a eventos embólicos, que muestran que se relacionan más con endocarditis infecciosa por SAMS. Es importante resaltar que múltiples estudios revelan el aumento de SAMR adquirido en la comunidad, hecho corroborado por esta investigación y que se convierte en una variable importante para evaluar en estudios posteriores. Es sustancial incluir en la terapia empírica de endocarditis infecciosa cobertura para SAMR hasta que se descarte este microorganismo. Se necesitan más estudios clínicos con mayor número de pacientes para conocer mejor el pronóstico de estas infecciones y los factores asociados a la resistencia a la meticilina en países como Colombia.

\section{Financiación}

Universidad Icesi y Fundación Valle del Lili.

\section{Conflictos de interés}

Ninguno.

\section{Bibliografía}

1. Hoen B, Duval X. Infective Endocarditis. N Engl J Med [Internet]. 2013 [cited 2018 Mar 12];368(15):1425-33. Disponible en: http://www.nejm.org/doi/10.1056/NEJMcp1206782

2. Sy RW, Kritharides L. Health care exposure and age in infective endocarditis: results of a contemporary population-based profile of 1536 patients in Australia. Eur Heart J [Internet]. 2010. Aug 1 [cited 2018 Mar 12];31(15):1890-7. Disponible en: http: //www.ncbi.nlm.nih.gov/pubmed/20453066

3. Ivan A, Noreña E, Asesores C, Arango A, Marín F, Beltran J. Caracterización clínica, microbiológica y ecocardiográfica de pacientes con Endocarditis infecciosa en la Fundación Cardioinfantil 2002-2012. [cited 2018 Mar 12]; Disponible en: http://repository.urosario.edu.co/bitstream/handle/10336/ 4976/1020722391-2014.pdf?sequence $=1$

4. Murdoch DR, Corey GR, Hoen B, Miró JM, Fowler VG, Bayer AS, et al. Clinical Presentation, Etiology, and Outcome of Infective Endocarditis in the $21^{\text {st }}$. Century. Arch Intern Med [Internet]. 2009. Mar 9 [cited 2018 Mar 12];169(5):463. Disponible en: http://www.ncbi.nlm.nih.gov/pubmed/19273776

5. Bouchiat C, Moreau K, Devillard S, Rasigade J-P, Mosnier A, Geissmann T, et al. Staphylococcus aureus infective endocarditis versus bacteremia strains: Subtle genetic differences at stake. Infect Genet Evol [Internet]. 2015. Dec 1 [cited 2018 Mar 12];36:524-30. Disponible en: https://www. sciencedirect.com/science/article/pii/S1567134815003524

6. Gallardo-García MM, Sánchez-Espín G, Ivanova-Georgieva R, Ruíz-Morales J, Rodríguez-Bailón I, Viñuela González $\mathrm{V}$, et al. Relationship between pathogenic, clinical, and virulence factors of Staphylococcus aureus in infective endocarditis versus uncomplicated bacteremia: a case-control study. Eur J Clin Microbiol Infect Dis [Internet]. 2016. May 7 [cited 2018 Mar 12];35(5):821-8. Disponible en: http://www.ncbi.nlm.nih.gov/pubmed/26951263

7. Hill EE, Peetermans WE, Vanderschueren S, Claus P, Herregods $M-C$, Herijgers P. Methicillin-resistant versus methicillin-sensitive Staphylococcus aureus infective endocarditis. Eur J Clin Microbiol Infect Dis [Internet]. 2008. Jun 26 [cited 2018 Mar 12];27(6):445-50. Disponible en: http://www.ncbi.nlm.nih.gov/pubmed/18224361

8. Eusse A, Atehortúa M, Vélez L, Bucheli V, Dallos C, Flores G, et al. Tratamiento quirúrgico de la endocarditis infecciosa. Rev Colomb Cardiol. 2014;21:52-7.

9. Bamberger DM. Bacteremia and endocarditis due to methicillin-resistant Staphylococcus aureus: the potential role of daptomycin. Ther Clin Risk Manag [Internet]. 2007. Aug [cited 2018 Mar 12];3(4):675-84. Disponible en: http://www.ncbi.nlm.nih.gov/pubmed/18472990

10. Echeverria R, Rozo J, Jaramillo C, Rodríguez C. Características epidemiológicas de la endocarditis infecciosa en la Fundación Clínica A, Shaio entre 1994-2001. Rev Colomb Cardiol. 2002;10:59-63.

11. Hase R, Otsuka Y, Yoshida K, Hosokawa N. Profile of infective endocarditis at a tertiary-care hospital in Japan over a 14-year period: characteristics, outcome and predictors for in-hospital mortality. Int J Infect Dis [Internet]. 2015. Apr [cited 2018 Mar 12];33:62-6. Disponible en: http://www.ncbi.nlm.nih.gov/pubmed/25576825

12. Salvador VBD, Chapagain B, Joshi A, Brennessel DJ. Clinical Risk Factors for Infective Endocarditis in Staphylococcus aureus Bacteremia. Texas Hear Inst J [Internet]. 2017. Feb [cited 2018 Mar 12];44(1):10-5. Disponible en: http://www.ncbi.nlm.nih.gov/pubmed/28265207

13. Habib G, Hoen B, Tornos P, Thuny F, Prendergast B, Vilacosta I, et al. Guidelines on the prevention, diagnosis, and treatment of infective endocarditis (new version 2009): The Task Force on the Prevention, Diagnosis, and Treatment of Infective Endocarditis of the European Society of Cardiology (ESC). Eur Heart J [Internet]. 2009. Oct 1 [cited 2018 Mar 12];30(19):2369-413. Disponible en: http://www.ncbi.nlm.nih.gov/pubmed/19713420

14. Akinosoglou K, Apostolakis E, Marangos M, Pasvol G. Native valve right sided infective endocarditis. Eur J Intern Med [Internet]. 2013. Sep [cited 2018 Mar 12];24(6):510-9. Disponible en: http: / / www.ncbi.nlm.nih.gov/pubmed/23369408

15. Yoon HJ, Choi JY, Kim CO, Kim JM, Song YG. A comparison of clinical features and mortality among methicillinresistant and methicillin-sensitive strains of Staphylococcus aureus endocarditis. Yonsei Med J [Internet]. 2005. Aug 31 [cited 2018 Mar 12];46(4):496-502. Disponible en: http://www.ncbi.nlm.nih.gov/pubmed/16127774

16. Murray PR, Rosenthal KS, Pfaller MA. Medical microbiology. $8^{\text {th }}$. ed Elsevier; 2015.

17. Carvalho SP de, Almeida JB de, Andrade YMFS, Silva LSC da, Oliveira AC de, Nascimento FS, et al. Community-acquired methicillin-resistant Staphylococcus aureus carrying SCC mec type IV and $\mathrm{V}$ isolated from healthy children attending public daycares in northeastern Brazil. Brazilian J Infect Dis [Internet]. 2017. Jul [cited 2018 Mar 12];21(4):464-7. Disponible en: http://www.ncbi.nlm.nih.gov/pubmed/28482183

18. Durack DT, Lukes AS, Bright DK. New criteria for diagnosis of infective endocarditis: utilization of specific echocardiographic findings. Duke Endocarditis Service. Am J Med [Internet]. 
1994. Mar [cited 2018 Mar 12];96(3):200-9. Disponible en: http://www.ncbi.nlm.nih.gov/pubmed/8154507

19. Ruotsalainen E, Sammalkorpi K, Laine J, Huotari K, Sarna S, Valtonen V, et al. Clinical manifestations and outcome in Staphylococcus aureus endocarditis among injection drug users and nonaddicts: a prospective study of 74 patients. BMC Infect Dis [Internet]. 2006. Sep 11 [cited 2018 Mar 12];6:137. Disponible en: http://www.ncbi.nlm.nih.gov/pubmed/16965625

20. Cosgrove SE, Sakoulas G, Perencevich EN, Schwaber MJ, Karchmer AW, Carmeli Y. Comparison of Mortality Associated with Methicillin-Resistant and Methicillin-Susceptible Staphylococcus aureus Bacteremia: A Meta-analysis. Clin Infect Dis [Internet]. 2003. Jan 1 [cited 2018 Mar 12];36(1):53-9. Disponible en: http://www.ncbi.nlm.nih.gov/pubmed/12491202
21. Alvarez CA, Barrientes OJ, Leal AL, Contreras GA, Barrero $\mathrm{L}$, Rincón $\mathrm{S}$, et al. Community-associated methicillin-resistant Staphylococcus aureus, Colombia. Emerg Infect Dis [Internet]. 2006. Dec [cited 2018 Mar 12];12(12):2000-1. Disponible en: http://www.ncbi.nlm.nih.gov/pubmed/17354345

22. Rincón S, Panesso D, Díaz L, Carvajal LP, Reyes J, Munita $J M$, et al. [Resistance to \&quot;last resort\&quot; antibiotics in Gram-positive cocci: The post-vancomycin era]. Biomedica [Internet]. 2014. Apr [cited 2018 Mar 12];34 Suppl 1(0 1):191-208. Disponible en: http://www.ncbi.nlm.nih.gov/ pubmed/24968051 